\title{
Decay of baryon inhomogeneities in an expanding universe
}

\author{
Pratik K. Das, Sovan Sau, Abhisek Saha, Soma Sanyal ${ }^{\mathrm{a}}$ (D) \\ University of Hyderabad, Prof. C.R. Rao Road, Hyderabad 500046, India
}

Received: 26 May 2021 / Accepted: 18 August 2021 / Published online: 15 September 2021

(C) The Author(s) 2021

\begin{abstract}
Baryon inhomogeneities are generated early in the universe. These inhomogeneities affect the phase transition dynamics of subsequent phase transitions, they also affect the nucleosynthesis calculations. We study the decay of the inhomogeneities in the early universe using the diffusion equation in the Friedmann-Lemaitre-RobertsonWalker metric. We calculate the interaction cross section of the quarks with the neutrinos, the electrons and the muons and obtain the diffusion coefficients. The diffusion coefficients are temperature dependent. We find that the expansion of the universe causes the inhomogeneities to decay at a faster rate. We find that the baryon inhomogeneities generated at the electroweak epoch have low amplitudes at the time of the quark hadron transition and hence will not affect the phase transition dynamics unless they are generated with a amplitude greater than $10^{5}$ times the background density. After the quark hadron transition, we include the interaction of the muons with the hadrons till $100 \mathrm{MeV}$. We find that large density inhomogeneities generated during the quark hadron transition with sizes of the order of $1 \mathrm{~km}$ must have amplitudes greater than $10^{5}$ times the background density to survive upto the nucleosynthesis epoch. This puts constraints on any models that generate these inhomogeneities
\end{abstract}

\section{Introduction}

Primordial cosmological fluctuations are an important part of modern cosmology as they link the current Cosmic Microwave Background Radiation (CMBR) data to the early universe. Initially the theory related to fluctuations was developed by Lifshitz [1]. Later on significant work has been done by Hawkings [2] and Bardeen [3]. In this work, we are interested in the decay of baryon density fluctuations or inhomogeneities generated in the early universe. Baryon density fluctuations can be generated in the electroweak phase

\footnotetext{
a e-mail: sossp.uoh@nic.in (corresponding author)
}

transition [4-6] as well as the QCD phase transition [7-9]. There are also various defect mediated mechanisms which generate these inhomogeneities at the electroweak scales [10-19]. Though baryon inhomogeneities have other roles to play in the evolution of the early universe, the primary method of constraining these inhomogeneities is by studying their effects on the Big Bang Nucleosynthesis calculations. The inhomogeneities generated at the QCD scales have a higher chance of surviving to the nucleosynthesis epoch, hence they have been studied more extensively. The baryon inhomogeneities generated in the electroweak epoch, have less chance of surviving till the nucleosynthesis epoch, hence they are often ignored. However it has been shown that baryon over densities generated in the electroweak epoch can definitely affect the quark hadron phase transition [20] and may also survive till the nucleosynthesis epoch [21]. One of the effect of these baryon over-densities is to delay the quark hadron phase transition in regions of the inhomogeneities. This, along with the fact that large scale inhomogeneities from the electroweak epoch may survive till the nucleosynthesis epoch and affect the abundances of the light elements makes it important to study the diffusion of particles in an inhomogeneity at the electroweak epoch.

The only detailed study of the evolution of non-linear sub horizon entropy fluctuations between $100 \mathrm{GeV}$ and 1 $\mathrm{MeV}$ was done in Ref. [21]. The entropy fluctuations that had been considered in that particular study did not necessarily come from baryon inhomogeneities only, the authors were not interested in the source of the entropy fluctuations. They had assumed certain amplitudes and length scales and evolved them with time. The evolution of the inhomogeneity was performed as a succession of pressure equilibrium states, that dissipate or expand due to neutrino heat transport. The neutrino contribution to the heat transport equation was used and after the quark hadron phase transition the neutron and proton diffusion were taken into account. They concluded that large scale entropy fluctuations at $100 \mathrm{GeV}$ could survive until the nucleosynthesis epoch and affect the 
nucleosynthesis calculations. The main difference between their study and our current study is that we look at baryon inhomogeneities generated at the electroweak scale in the diffusive limit taking into consideration both the quark and the lepton diffusion. We will be studying sub horizon scale baryon density fluctuations. Studies have already shown that baryon inhomogeneities can be generated in the electroweak scale. Generally, they have an amplitude of around $10^{4}$ times the background baryon density. Since, the baryon number is carried by the quarks at these high temperatures, the baryon inhomogeneities at these scales will primarily mean an excess of the number density of quarks in a certain region. These quarks diffuse out by colliding with the leptons, which are the electrons, muons and the neutrinos. In this study we focus on obtaining the diffusion coefficient of these quarks as they move through the plasma. The diffusion coefficient is then used in the particle diffusion equation to study the decay of baryon inhomogeneities in an expanding universe.

The diffusion equation has been studied in the early universe for the case of baryon diffusion in the hadronic phase [22-24] when the baryon number is carried by the neutrons and the protons. Here, for the first time, we use it to study, baryon diffusion in the quark gluon plasma phase. Since we are using the diffusion equation pertaining to an expanding universe, we also extend our study to the QCD scales. We do find that the expansion term makes a significant difference in the decay of the inhomogeneities. We find that in a static universe the decay rate is slow, while in an expanding universe, the inhomogeneities decay much faster. In the electroweak case, the particles that we consider are the quarks, muon, electrons and the neutrinos. For the QCD epoch, we have the hadrons which carry the baryon number. Generally, the inhomogeneities which are generated at the QCD epoch have higher amplitude and sizes than those generated in the electroweak epoch [25]. Hence we consider these two cases separately.

In this work, we only look at sub horizon scale fluctuations. We also assume that the size of the fluctuations are larger than the mean free path of the relevant particles. The diffusion equation, is re-written in the Friedmann-LemaitreRobertson-Walker (FLRW) metric. We neglect baryon number violating processes at the temperatures around $200 \mathrm{GeV}$ and assume that the total baryon number density is conserved throughout our calculations. All the baryon density fluctuations we consider are assumed to be Gaussian fluctuations.

The chief motivation of understanding the decay of the baryon inhomogeneities is the different signatures of Inhomogeneous Big Bang Nucleosynthesis (IBBN) that have been predicted [26]. An inhomogeneous BBN will result from patches of region where there is a baryon inhomogeneity $[27,28]$. However, all the study so far has concentrated only on inhomogeneities generated in the quark hadron phase transition. The only study for entropy fluctuations generated in the electroweak epoch has indicated that there will be no significant decay during the period prior to the quark hadron phase transition. We were motivated to see whether this result will hold for baryon inhomogeneities also. We however, find that this result does not hold for baryon inhomogeneities. The inhomogeneities decay rapidly during this period and they decrease by about three orders of magnitude. Since some of the inhomogeneities generated in the electroweak epoch have an amplitude of only $10^{4}$, these will decay and have no effect on the quark hadron phase transition or the nucleosynthesis epoch. We also study the decay of the inhomogeneities after the quark hadron phase transition. Though these will undergo significant decay, some of them might survive till the nucleosynthesis epoch. The BBN can then be used to constrain the models that generate these fluctuations [29-32].

In Sect. 2, we discuss the amplitude and size of the baryon inhomogeneities that are of interest to us. In Sect. 3 we discuss the diffusion equation in the FLRW metric, in Sect. 4, we obtain the diffusion coefficient in the quark gluon plasma phase. Since we take into account the scattering of the quarks with electrons, muons and neutrinos, we have divided this section into three subsections. In Sect. 5 we present the numerical results of the decay of the baryon overdensities between the temperature $200 \mathrm{GeV}$ and $200 \mathrm{MeV}$. This is the period after the electroweak phase transition and before the quark hadron transition. In Sect. 6, we discuss the decay of the inhomogeneities after the quark hadron transition. Inhomogeneities which survive upto the nucleosynthesis period will affect the light element abundances. In Sect. 7, we summarize our work and present our conclusions.

\section{Baryon inhomogeneities in the early universe}

There are several ways in which baryon over densities can be generated in the early universe. Topological defects such as electroweak strings are unstable and generate baryon number when they decay [33-38]. These give rise to local baryon density fluctuations. The scale of these fluctuations will be given approximately by the bubble nucleation distance at the electroweak scale. Inhomogeneities may thus be generated by these strings over small length scales $\left(10^{-10} \mathrm{cms}\right)$. These inhomogeneities have a large amplitude and consequently diffuse out. Other than the electroweak strings, superconducting strings are also capable of generating baryon inhomogeneities over small lengthscales $[39,40]$. The most detailed study of baryon inhomogeneity generation, their amplitude and size was done in Ref. [5]. The lengthscales of the inhomogeneities generated were about $10^{-3} \mathrm{~cm}$ and they had an amplitude of $10^{4}$ over the background baryon density. We will not be considering any specific model during this epoch, our focus would be to see if these baryon inhomogeneities can survive at least upto the QCD epoch. We have taken Gaus- 
sian fluctuations to represent the baryon inhomogeneities. These are characterised by the amplitude $(A)$ and the full width at half maxima $(b)$. We have kept the $A \sim 10^{3}$ as it is expected that the baryon inhomogeneities at the electroweak scale are not too high. The variance basically determines the radius of the inhomogeneities. The amplitude of the baryon inhomogeneities represent the ratio of the number density of the baryons in the inhomogeneity $\left(n_{b}^{\prime}\right)$ over the background number density $\left(n_{b}\right)$. So that we have $A \sim \frac{n_{b}^{\prime}}{n_{b}}$. The pressure of the inhomogeneities can be related to the amplitude. The pressure in the quark phase $\left(p_{q}\right)$ is given by,

$p_{q}=\frac{7}{4} N_{q} a T^{4}+9 N_{q}^{-1} \frac{n_{b}^{2}}{T^{2}}$

Here, the second term on the right gives the contribution to the pressure from the baryon number density. $N_{q}$ is the number of relativistic quarks at temperature $T$ and $a=\frac{\pi^{2}}{30}$. The inhomogeneities reach pressure equilibrium very fast and a small temperature difference exists between the inhomogeneity and the background. The temperature difference between the background and the inhomogeneity has been calculated in [20] and is given by,

$\frac{\delta T}{T}=-3 \times 10^{-19} \times A^{2}$

So unless the amplitude of the inhomogeneity is quite large it does not lead to significant temperature fluctuations. So we see that the amplitudes of the inhomogeneities are an important measure of the consequences of baryon inhomogeneities in the early universe.

For the inhomogeneities at the QCD scale we have taken a higher amplitude. This is because there is a greater probability of generating large over densities at the QCD scale [41]. Inhomogeneities can be generated by moving cosmic strings [25], collapsing Z(3) domain walls [42,43] and inhomogeneous nucleation of bubbles in a first order phase transition [44]. Inhomogeneities can be spread over an area of one meter and can have amplitudes of the order of $10^{12}-10^{13}$ through these various mechanisms. Larger inhomogeneities may also be generated but they will have lower amplitudes. Again we do not focus on the mechanism that will lead to the generation of these baryon inhomogeneities, we only study the inhomogeneities over different ranges for an approximate amplitude of $10^{4}$ times the background baryon density. Diffusion and decay of baryon inhomogeneities in the QCD epoch has been studied before. In a previous work [24] we have worked out the decay of baryon inhomogeneities using the diffusion equation but ignoring the expansion of the universe. In this work, we redo the multiparticle diffusion that we had done previously in [24] for the case of an expanding universe. We find that the expanding universe has a significant contribution to the decay of the large scale inhomogeneities. Whereas in the previous work we had considered inhomo- geneities whose sizes are much smaller than the horizon size, in this work we consider large scale baryon inhomogeneities and see how they decay in the expanding universe.

\section{The diffusion equation in the FLRW metric}

In this section, we briefly describe the diffusion equation in the FLRW metric, in terms of baryon diffusion in the early universe. The FLRW metric for the flat universe is defined by,

$d s^{2}=c^{2} d t^{2}-a^{2}(t) d \mathbf{r}^{2}$

Here $a(t)$ is the scale factor of the expanding universe and $\mathbf{r}$ is the spatial coordinate. This is the comoving distance in an expanding universe. Consider a region of the universe with an inhomogeneity given by $n(\mathbf{r}, t)$. As time evolves, the particles in the over dense region tend to move towards the lesser dense region to restore equilibrium and a particle flux is generated. In this case, we consider the diffusion to be isotropic. The local observer then sees the particle flux as,

$j_{k}=-D(t) \frac{\partial}{\partial x^{k}} n(\mathbf{r}, t)$

The diffusion coefficient $D(t)$ depends on the scattering cross section and the velocity of the particles. Since there are different kinds of particles in this plasma, we are dealing with multi-particle diffusion here. The conservation of current gives us,

$\frac{\partial}{\partial x^{\mu}}\left(\sqrt{g} j^{\mu}\right)=0$

Here $\sqrt{g}=a^{3}(t)$ and using the definition of the Hubble parameter as $H(t)=\frac{\dot{a}(t)}{a(t)}$, the diffusion equation can be written as,

$\frac{\partial}{\partial t} n(\mathbf{r}, t)+3 H(t) n(\mathbf{r}, t)-\frac{D(t)}{a^{2}} \nabla^{2} n(\mathbf{r}, t)=0$

This is the diffusion equation that we will solve numerically for a time dependent diffusion coefficient. As mentioned before, the diffusion coefficient depends on the scattering cross section of the particles. Hence it is different at different temperatures. Since the scattering cross sections are obtained in terms of temperatures, we use the time-temperature relation in the radiation dominated universe to convert our time to temperature.

$t=\frac{\left(0.95 \times 10^{10}\right)^{2}}{T^{2}}$

Here $t$ is in secs and $T$ is in Kelvin. We then solve the diffusion equation in the FLRW metric numerically over the entire range of temperature from $200 \mathrm{GeV}$ to $200 \mathrm{MeV}$. In the next section, we first present the details of calculating the diffusion coefficient in the quark phase. 


\section{Diffusion in the electroweak scale}

We start by studying particle diffusion in the electroweak scale. The inhomogeneities are formed at $200 \mathrm{GeV}$ during the electroweak phase transition. So the diffusion of particle will start around the same time. During this epoch the most abundant particles are the quarks, electrons, muons and the neutrinos. Out of all these, it is the quarks which carry the baryon number. So the baryon over-densities would predominantly have a larger density of quarks as compared to the background number density. As the quarks diffuse out of the inhomogeneities trying to reach an equilibrium state they will collide with the electrons, muons and the neutrinos. Here we take two cases depending on the mass of the particles. This is because the quarks are lighter than the muons but heavier than the electrons. So as the quarks move through the muons, we have a lighter gas diffusing into a gas of heavier particles but as the quarks diffuse through the electrons and neutrinos, we have a heavier particle diffusing through a lighter gas [45]. Since we are not going into the detailed transport equation of the particles, we choose a distribution function for the particles of the light gas.

In the first case, for the quarks moving through the muons, the diffusion coefficient is given by,

$D=\frac{1}{3 N}\left\langle\frac{v}{\sigma_{t}}\right\rangle=\left(\frac{T}{\pi m}\right)^{1 / 2} \frac{2^{3 / 2}}{3 \sigma_{t}}$

In the second case, for a heavier particle moving through a lighter fluid, to obtain the diffusion coefficient, we have to first compute the mobility of the particle in the background fluid. If the velocity of the particle is $\mathbf{v}$, then the mobility $b$ is related to the external force (f) by, $\mathbf{v}=b \mathbf{f}$ and the diffusion coefficient is given by,

$D=b T$

We assume the distribution of particles to be Maxwellian, then the mobility of the particles is given by,

$b^{-1}=\frac{16 \pi}{T} \int \frac{p^{2} d p}{3 h} v p^{2} \sigma_{t} e^{-E / T}=\frac{16 \sigma_{t} m^{2} t^{2}}{3 \pi^{2}}$.

Here $\sigma_{t}$ is the scattering cross-section, $m$ is the mass of the particle. Once the scattering cross section is known, substituting it in the expression for $b$ would enable us to obtain the diffusion coefficient $D$. Since the scattering cross-sections are temperature dependent, the diffusion coefficient too would be temperature dependent. To find the diffusion coefficient at these temperatures we therefore, obtain the scattering cross section of the quarks with the leptons. In the next subsections, we will calculate the different scattering cross section for the different interactions.

\subsection{Quark-electron scattering}

We start with the motion of quarks through the electron gas. For this we need to find the scattering cross section for the $e^{-} e^{+} \longrightarrow q \bar{q}$ interaction. The differential cross section is given by,

$\frac{d \sigma}{d \Omega}=\frac{Q_{f}^{2} \alpha^{2}}{2 s}\left(\frac{u^{2}+t^{2}}{s^{2}}\right)$

Here $\alpha \sim 10^{-2}$ is the fine structure constant and $Q_{f}$ is the momentum transfer in this interaction. The variables $u, t$ and $s$ are the Mandelstam variables. This gives,

$\sigma_{t}=\frac{Q_{f}^{2} \alpha^{2}}{2 s} \int\left(\frac{u^{2}+t^{2}}{s^{2}}\right)(1-\cos \theta) d \Omega$

The total scattering cross section can be obtained after integrating over the solid angle. The numerical value can be obtained once the energy scale of the colliding particles is known. Since we are working around the electroweak scale, the colliding energy of the particles are also in the $\mathrm{GeV}$ range. The mobility factor is thus given by,

$b^{-1}=\frac{2 \sigma_{t} m^{2}}{3 \pi^{2}}\left[8 T^{2}\left(1-e^{-E / T}\right)-2 E(2 E+4 T) e^{-E / T}\right]$

The diffusion coefficient can be calculated numerically after obtaining the mobility at various temperatures.

\subsection{Quark-neutrino scattering}

Neutrinos do not have any charges, they have weak interactions. Though there are different flavors of quarks as well as neutrinos, since we only need order of magnitude estimations, we just consider,

$\sigma_{t}=\frac{G_{F}^{2} \hat{s}}{\pi}$

Here $G_{F}$ is the Fermi constant given by, $G_{F}=1.166 \times$ $10^{-5} \mathrm{GeV}^{-2}$. Numerically, the cross section turns out to be $\sigma_{t}=17.2 \times 10^{-42} \mathrm{~cm}^{2} \times \frac{E_{v}}{\mathrm{GeV}}[46]$. Though we are working at very high temperatures in the $\mathrm{GeV}$ scale, the value of the diffusion coefficient is difficult to handle numerically with this value of $\sigma_{t}$. For the numerical calculation we therefore rescale the variables suitably to obtain a stable numerical solution.

\subsection{Quark-muon scattering}

In both the previous cases we had a heavier particle moving through a lighter gas of particles, however the scenario changes considerably when we consider the quarks moving through a gas of muons. For the $\mu^{-} \mu^{+} \longrightarrow q \bar{q}$, though the expression for the interaction cross sections are similar to the 
electrons, but here the quark is the lighter particle which is moving through a heavier gas of particles (the muons). This means that, the diffusion coefficient is given by Eq. (8),

$$
\begin{aligned}
D= & \left(\frac{1}{2 \pi}\right)^{\frac{3}{2}}\left(\frac{s^{\frac{1}{2}}}{Q_{f} \alpha}\right)^{2}\left(\frac{1}{m T}\right)^{\frac{1}{2}} \\
& \times\left[2 T\left(1-e^{-E / T}\right)-2 E e^{-E / T}\right]
\end{aligned}
$$

We have the total cross section given by,

$\sigma_{t}=\frac{4 \pi Q_{f}^{2} \alpha^{2}}{3 s}$

In all these cases, we determine the diffusion coefficient at different temperatures numerically. Similar to the quark neutrino cross section, even at such high temperatures, the diffusion coefficient are numerically very large quantities hence for all the different cases we need to do some scaling to obtain numerically stable solutions. This we have done by scaling the energy appropriately so that the value of the diffusion coefficient is of reasonable orders of magnitude. While plotting we have plotted only the amplitude at the different length scales depending on the temperature. The amplitude is dimensionless as it is the ratio of the enhanced density to the background density $\left(\frac{n_{B}^{\prime}}{n_{B}}\right)$. Here $n_{B}^{\prime}=\Delta n_{B}+n_{B}$ and $n_{B}$ is the average baryon density at that temperature and $\Delta n_{B}$ is the increase in the baryon density in the inhomogeneity. Thus the rescaling does not affect the change in the overdensity that we are interested in. Only, the length scales are changed appropriately to reflect the decrease in the temperature.

\section{Decay of inhomogeneities in the quark gluon plasma phase}

For the numerical study, we have used the finite difference method for the second order space derivative and an explicit forward Euler approximation for the first derivative in time. The Courant-Friedrichs-Loewy coefficient changes with temperature but care is taken so that it never exceeds 0.5 for maintaining the stability of the program. Here we have considered the solution of Eq. (6) to be spherically symmetric and the solution will thus depend upon the radius $r$ which we consider to be the length scale of the inhomogeneity. The origin is taken to be at the centre and the particles diffuse out causing the radius of the inhomogeneity to increase. The maximum value of the radial lattice is the horizon size. As we are working in the FLRW metric, the universe size will also increase with decrease in temperature. This feature is incorporated in our program by increasing the maximum size of the radius as time increases and temperature decreases. We have kept the minimum step size fixed at $d r=0.01$. Time is represented by temperature and the maximum lattice size is therefore calculated at each temperature before the $r$ loop.
The relationship is given by, $r_{\max }=\frac{314.01155}{T}$. For both the electroweak and the QCD case, we start with a Gaussian fluctuation given by $f(r)=A e^{\left(-\frac{r^{2}}{2 b^{2}}\right)}$. The amplitude $A$ and the variance $b$ are different in the two epochs. The amplitudes are different as the generation of baryon inhomogeneities in the two epochs are different and the variance are different as length scales in the two epochs are different.

We now look at baryon inhomogeneities generated during the electroweak phase transition. In Refs. [5,6], it is shown that a strong first order phase transition generates a radially symmetric baryon inhomogeneity whose amplitude depends upon the ratio of the minimum and maximum bubble wall velocity. They have shown that baryon inhomogeneities having amplitudes of the order of $10^{2}$ are generated. Though we are not emphasizing that these are the inhomogeneities we are interested in, they do provide a measure of the possible magnitude of baryon inhomogeneities generated at the electroweak scale. So we consider the amplitude of the inhomogeneities generated in the electroweak epoch to be of the order of $10^{3}$. We are also considering inhomogeneities whose decay will be affected by the expanding universe. The horizon at these temperatures is of the order of $1 \mathrm{~cm}$ [47]. The size of the inhomogeneities we have considered are always less than the horizon size. However we do not want them to be so small that their lengthscale is negligible in comparison to the radius of the universe. So basically we have looked at inhomogeneities which have a radius of less than a millimeter. We have presented results for inhomogeneities having a radius of $0.03 \mathrm{~cm}$. Larger inhomogeneities (with radius closer to 1 $\mathrm{cm}$ ) will also decay in a similar way but it is rather difficult to present the graphs in the same plot due to the vast change in the radius. Hence for presenting our results we have chosen the radius of the initial inhomogeneity to be about $0.03 \mathrm{~cm}$. The diffusion coefficients can be obtained numerically, but the problem is that they vary considerably in their numerical values. This indicates that the particle content in the inhomogeneity would ultimate define how they decay. Though there are multi particles present in the plasma, we do not go for multi particle diffusion as it becomes numerically quite challenging. We look at each of these interactions separately and see how much each of them contributes to the decay of the baryon inhomogeneity. We believe this will give us some idea of how the baryon inhomogeneity decays in this temperature range.

There are several challenges in the electroweak epoch. The biggest challenge is the large change in the horizon size between $200 \mathrm{GeV}$ and $200 \mathrm{MeV}$. At $200 \mathrm{GeV}$, the horizon is of the order of $1 \mathrm{~cm}$ while at $200 \mathrm{MeV}$ the horizon is of the order of $10 \mathrm{kms}$. We divide this into two parts. We evolve the inhomogeneity from $200 \mathrm{GeV}$ to about $1 \mathrm{GeV}$ and then again from $1 \mathrm{GeV}$ to $200 \mathrm{MeV}$. Interestingly, we find that the inhomogeneity decays considerably during this period 


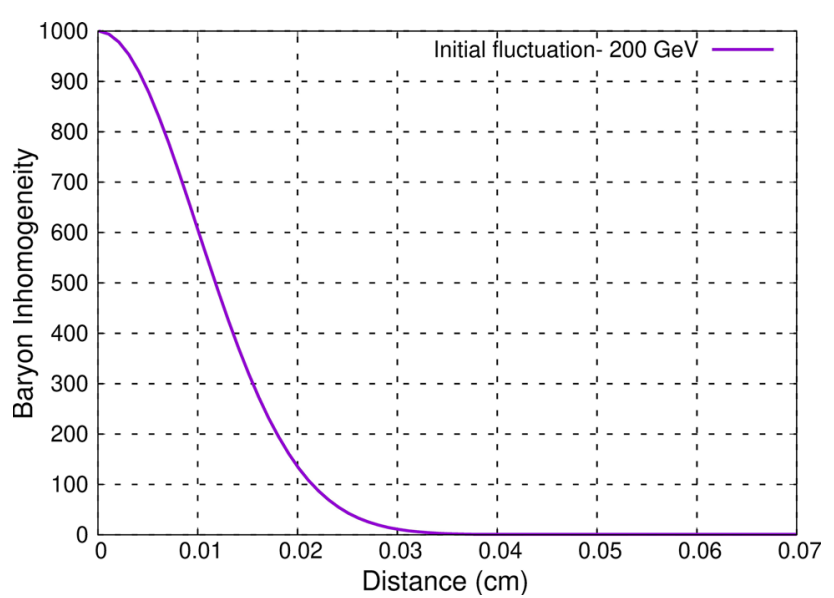

Fig. 1 The initial fluctuation at $200 \mathrm{GeV}$ in the linear scale

depending on the particle interactions being considered. We find that on the log scale, the amplitude decays quite rapidly irrespective of the interactions considered. This means that low amplitude inhomogeneities will be completely wiped out before the quark hadron phase transition.

Let us first look at the decay of the inhomogeneities between $200 \mathrm{GeV}$ and $1 \mathrm{GeV}$. We give the figure of the initial fluctuation in Fig. 1. The graph is plotted in the linear scale, however in the rest of the paper, the results are plotted in the $\log -\log$ scale instead of the linear scale. In the logarithmic scale, the function appears to end abruptly as the zero is not defined on this scale. Since the lattice size of the simulation also changes with temperature, the data for the higher temperatures are for a smaller radius (horizon) size, while the data for lower temperatures reflects the larger radius of the horizon. In the graphs, we have focussed on the decrease in the amplitude of the inhomogeneity rather than the increase in it's size. Even with the increase in size, the fluctuations always remain within the horizon.

In the electroweak epoch, the diffusion coefficients differ considerably as they are dependent on the temperature and this is quite a large temperature range. So we look at the decay of the inhomogeneities for different interactions separately. For all the different figures, we have the baryon inhomogeneity $\left(\frac{n_{B}^{\prime}}{n_{B}}\right)$, on the y axis and the radial length scale (denoted by distance) on the x-axis. In Fig. 2, we see the decay due to the quarks moving through the electrons. The initial fluctuation is taken at $200 \mathrm{GeV}$ and the final is taken at $17 \mathrm{GeV}$.

As we see the peak of the inhomogeneities goes down by more than three orders of magnitude. The inhomogeneity also spreads out. If we plot the solution upto the maximum radial distance, then for the initial curve we observe an abrupt cut off. The abrupt cut off is an artefact of plotting the solution in the log-log scale. The same plot in the linear scale resembles Fig. 1 but with a reduced peak and larger variance. However,

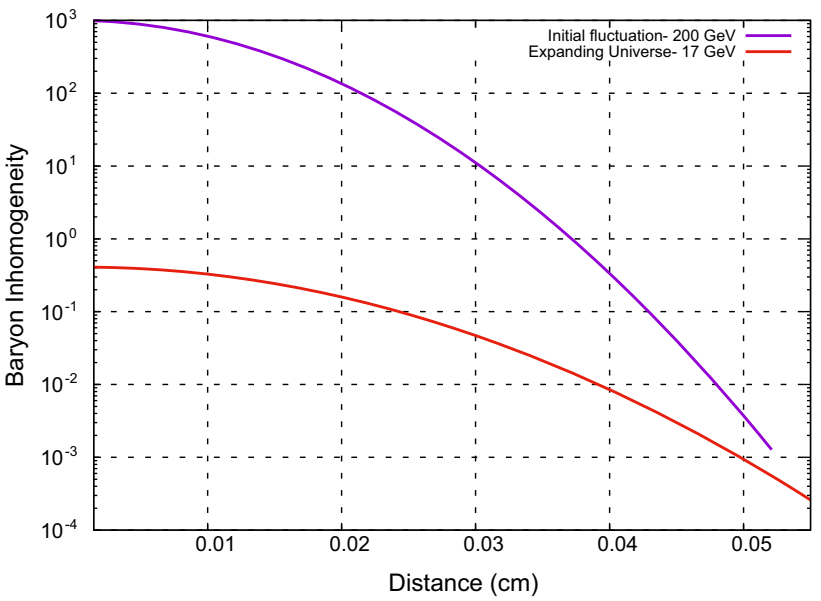

Fig. 2 The decay of the fluctuation is shown in logscale between 200 and $17 \mathrm{GeV}$ as the quarks moves through a sea of electrons. The fluctuation is smaller at higher temperatures and spreads out further as temperature decreases

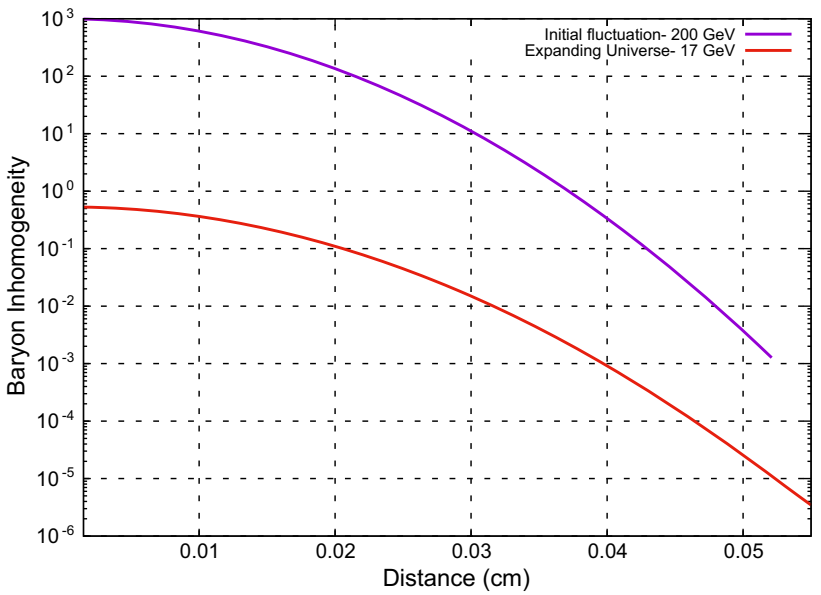

Fig. 3 The decay of the fluctuation is shown in logscale between 200 and $17 \mathrm{GeV}$ as the quarks are predominantly surrounded by neutrinos. The fluctuation is smaller at higher temperatures and spreads out further as temperature decreases

since there is a considerable decrease in the amplitude, it is not possible to show both the graphs in a linear plot. The decay is similar in the case when the surrounding particles are muons and neutrinos. We have used a different scaling for the neutrinos but as mentioned before the scaling will not affect the relative decay of the amplitude. Though the final amplitude is lower in the case of the neutrinos, the order of magnitude is similar. Since all the graphs have similar decay in orders of magnitude, we have only shown selected graphs. Figure 2, shows the decay due to the motion of the quarks through the electrons while Fig. 3 shows the decay due to the motion of the quarks through the neutrinos. Since the plasma at those high temperatures is predominantly dominated by electrons, our results clearly show that the baryon inhomogeneities decay by about three orders of magnitude in the 


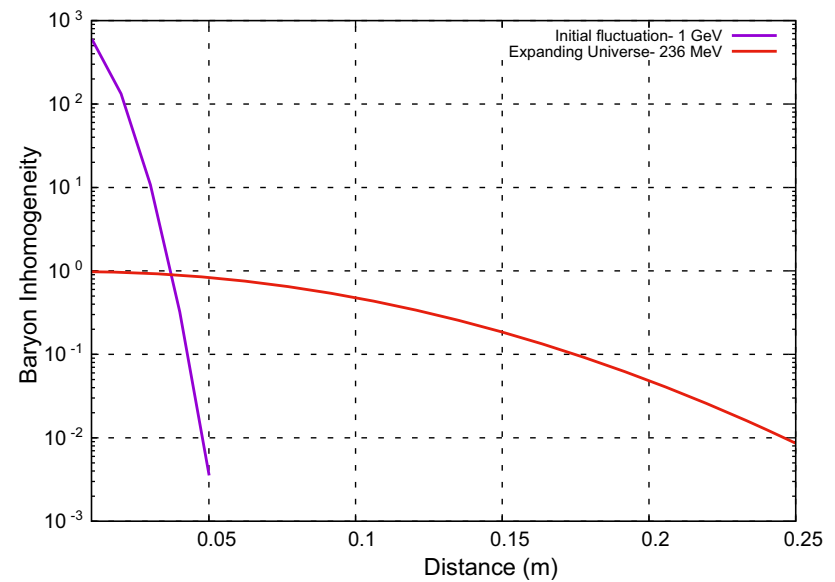

Fig. 4 The decay of the fluctuation is shown in log scale between 1 $\mathrm{GeV}$ and $236 \mathrm{MeV}$ for a plasma where a quark is moving through a sea of electrons

high temperature $\mathrm{GeV}$ range. This is true, even if there are a large number of muons and neutrinos in the plasma.

We now look at the decay of the inhomogeneities between the temperatures $1 \mathrm{GeV}$ to $200 \mathrm{MeV}$. We find that the order of magnitude decay is again quite large for the three diffusion coefficients. The individual numbers vary but we plot only the order of magnitude estimates as before. In Fig. 4, we find that the inhomogeneity has decreased by three orders of magnitude. In the case of Fig. 4, the initial fluctuation is at $1 \mathrm{GeV}$ while the final is plotted at $236 \mathrm{MeV}$. We find that the curve flattens out considerably. The radial distance upto which the inhomogeneity persists is actually more than what is shown in the plot. Again we find that the initial inhomogeneity appears to have a sudden cut off around $0.05 \mathrm{~cm}$. This is again due to the $\log \log$ nature of the plot. The size of the inhomogeneity is also much smaller initially. Since the maximum radius of the lattice is smaller at higher temperatures, the initial fluctuation looks very narrow and sharp when plotted at a later time. This makes the shape of the fluctuation different at different times. Not only are the particles diffusing away from the centre of the inhomogeneity, the maximum size of the one dimensional lattice is also increasing with time.

For the case of the quarks moving through a large number of muons, the amplitude decay is less than the decay in the case when the particles surrounding the quarks are the electrons. However, the decay is still quite significant. For the neutrinos, again we have the inhomogeneity decaying by three orders of magnitude. So independent of the particle distribution in the plasma, the amplitude of the inhomogeneities goes down significantly. In Fig. 5, we have the quarks moving in a region of muons while in Fig. 6, the quarks move through the neutrinos. As is seen from the plot, the inhomogeneity decays much faster in the presence of neutrinos and quickly reaches an amplitude of $10^{-2}$. Compared to the initial amplitude, this is negligible and can be considered to be

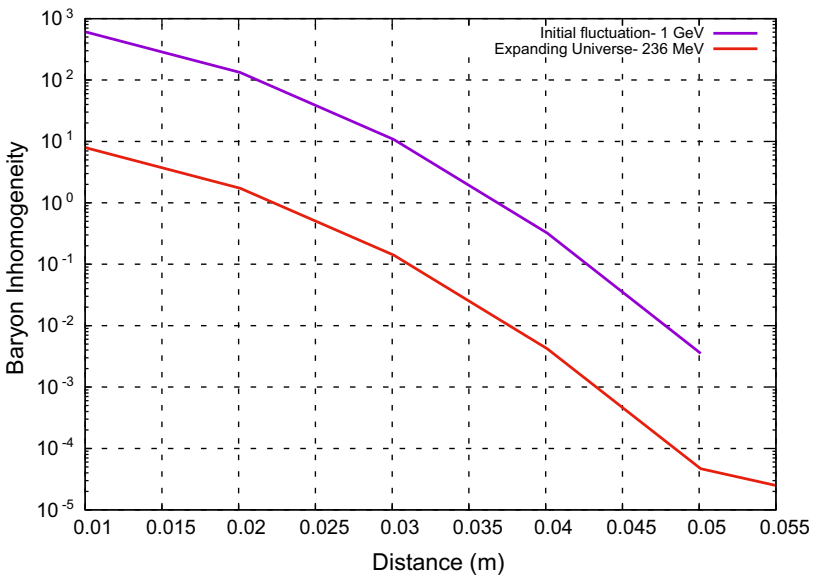

Fig. 5 The decay of the fluctuation is shown in log scale between 1 $\mathrm{GeV}$ and $236 \mathrm{MeV}$ for a plasma where a quark is moving through muons

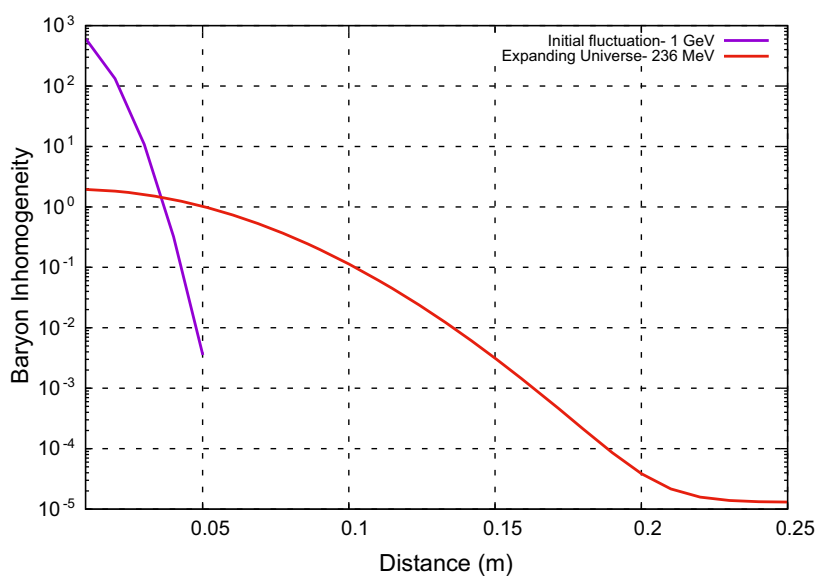

Fig. 6 The decay of the fluctuation is shown in $\log$ scale between 1 $\mathrm{GeV}$ and $236 \mathrm{MeV}$ for a plasma where a quark is moving through neutrinos. Since the inhomogeneity decays faster here, as the magnitude goes down, the radial distance increases. The full curve at $236 \mathrm{MeV}$ is not given, it is cut off at some point where baryon inhomogeneity is close to 0.01 . Compared to the initial fluctuation which had an amplitude of $10^{3}$, this can be taken to be zero

zero. That is why in Fig. 6 the plot is cut off at a distance $25 \mathrm{~cm}$. Beyond this point, the inhomogeneity gradually goes down to zero.

We thus find that the inhomogeneity decays by three orders of magnitude between 200 and $1 \mathrm{GeV}$, and again decays by at least two orders of magnitude between $1 \mathrm{GeV}$ to 200 $\mathrm{MeV}$. This means that any inhomogeneity generated at the electroweak epoch needs to have an amplitude greater than $10^{5}$ times the background density to survive till the quark hadron transition. Thus if we had an inhomogeneity at the electroweak scale with an amplitude less than $10^{5}$, it would be completely wiped out before the quark hadron transition. 


\section{Decay of inhomogeneities in the hadronic phase}

We have studied the decay of inhomogeneities in the hadronic phase previously [24], however in that case we were interested in specific inhomogeneities generated by $\mathrm{Z}(3)$ domain walls whose size was much smaller compared to the horizon size. The expansion of the universe was ignored in those cases. We are now interested to see the decay of larger inhomogeneities in the hadronic phase. As mentioned before, inhomogeneities generated during or after the quark hadron phase transition are not only larger in amplitude but they may also be larger in size. Consequently, the decay of these inhomogeneities would be affected by the expanding universe. The plasma during this period consists of the muons, neutrons, protons. electrons and neutrinos. In our previous work, we had shown that the presence of muons enhances the diffusion coefficient of the neutrons/protons, however we had not factored in the expansion of the universe in the previous work. This time we use the diffusion equation for the expanding universe and find that the expansion of the universe causes the inhomogeneities to decay much faster. In the current section, we briefly describe the diffusion coefficient in the hadronic phase and then proceed to present the results of the decay of the inhomogeneities in the hadronic plasma. In this case, the size of the inhomogeneities is taken to be of the of the order of kilometers as the horizon size is around $10 \mathrm{Kms}$ after the quark hadron transition.

As we have mentioned, the calculation of the diffusion coefficient will depend on which particle is moving through the plasma. The baryon number is carried by the neutrons and the protons, hence here we will be considering the motion of a heavier particle through a lighter gas. The heavier particle is the neutron or the proton, while the lighter gas is a gas of electrons and neutrinos. The muons only play a role till $100 \mathrm{MeV}$. We thus have to use Eq. (9) and the scattering cross-section of the neutrons with the electron-positron gas to obtain the diffusion coefficient of the neutrons in the electron positron gas. The scattering cross section is given by,

$\frac{d \sigma}{d \Omega}=\frac{\alpha^{2} \kappa^{2} q^{2}}{16 M^{2} E^{2} \sin ^{4}(\theta / 2)} \frac{E^{\prime}}{E} \times\left[1+\sin ^{2}(\theta / 2)\right]$

Here $\theta$ is the scattering angle, while $E$ is the electron energy before the scattering and $E^{\prime}$ is the electron energy after the scattering. The values of $E$ and $E^{\prime}$ depend on the temperature of the surrounding plasma. The transport cross section $\sigma_{t}$, is given by

$\sigma_{t}=\int \frac{d \sigma}{d \Omega}(1-\cos \theta) d \Omega$
We can then substitute the scattering cross-section in the transport cross section to obtain,

$\sigma_{t}=3 \pi\left[\frac{\alpha \kappa}{M}\right]^{2}$

The diffusion coefficient is obtained by substituting the expression for the transport cross-section and we get,

$D_{n e}=\frac{M^{2}}{32 m^{3}} \frac{1}{\alpha \kappa^{2}} \frac{e^{1 / T}}{T f(T)}$.

Here $M$, is neutron mass, $m$ is electron mass and $\kappa=-1.91$ is the anomalous magnetic moment. The temperature in this case is dimensionless as it is scaled by a factor of $m_{e} c^{2}$. Finally, the function $f(T)$ is given by, $f(T)=1+3 T+3 T^{2}$.

Similar to the neutron-electron cross section, we can obtain the nucleon-muon scattering cross-section too. We have assumed that the heavy neutron particle is moving through a muon-antimuon gas. The mobility of the neutron is then given by the force on the neutron due to the gas. This force is given by the interaction cross section. The differential scattering cross-section is given by,

$$
\begin{aligned}
\frac{d \sigma}{d \Omega}= & \frac{\alpha^{2} \kappa^{2} q^{2}}{8 M^{2} E^{2} \sin ^{4}(\theta / 2)} \frac{1}{1+2 E \sin ^{2}(\theta / 2) / M} \\
& \times\left[\frac{\cos ^{2}(\theta / 2)}{1-q^{2} / 4 M^{2}}\left(\frac{q^{2}}{4 M^{2}}-1\right)-2 \sin ^{2}(\theta / 2)\right]
\end{aligned}
$$

Here we have assumed that the muon energy and mass are less than the neutron mass. The cross-section calculation is simplified by this assumption and we can write the crosssection as,

$\frac{d \sigma}{d \Omega} \approx K \frac{\alpha^{2} \kappa^{2}}{4 M^{2}}\left[1+\operatorname{cosec}^{2}(\theta / 2)\right]$

All the constants are replaced by a single constant $K=\frac{1}{2}$. We then substitute the cross section in the diffusion constant. Finally, the diffusion coefficient is given by,

$D_{n \mu}=\frac{M^{2}}{32 m_{\mu}^{3}} \frac{1}{\alpha \kappa^{2}} \frac{e^{1 / T^{\prime}}}{T^{\prime} f\left(T^{\prime}\right)}$

Here $T^{\prime}=\frac{T}{m_{\mu} c^{2}}$. After obtaining both $D_{n e}$ and $D_{n \mu}$, we calculate the total diffusion coefficient of the neutron moving through the plasma.

Apart from the neutron, we need to find the diffusion coefficient of the proton moving through the electron positron gas too. For proton-electron scattering, the Coulomb force has to be taken into consideration. The scattering cross section for the proton and electron is then given by,

$$
\frac{d \sigma}{d \Omega}=\frac{\alpha^{2} m_{e}^{2}}{4 k^{4} \sin ^{4}(\theta / 2)}\left[1+\frac{k^{2}}{m_{e}^{2}} \cos ^{2}(\theta / 2)\right]
$$


We can obtain the transport cross section from these equations,

$\sigma_{t}=4 \pi \alpha^{2}\left[\frac{E_{e} h}{2 \pi k^{2}}\right]^{2} \ln \left(\frac{2}{\theta_{0}}\right)$

where $\theta_{0}$ is the minimum scattering angle. Substituting all the previous equations, we get the diffusion coefficient as,

$D_{p e}=\frac{3 \pi}{8 \alpha^{2} \ln \left(\frac{2}{\theta_{0}}\right)}\left[\frac{h}{2 \pi m_{e}}\right] \frac{T e^{1 / T}}{f(T)}$.

Since the muons also constitute a significant part of the plasma till $100 \mathrm{MeV}$, we calculate the proton muon cross section too. The differential cross section is given by,

$$
\begin{aligned}
\frac{d \sigma}{d \Omega}= & \frac{\alpha^{2}}{4 E^{2} \sin ^{4}(\theta / 2)} \frac{1}{1+2 E \sin ^{2}(\theta / 2) / M} \\
& \times\left[\left(1-\frac{\kappa^{2} q^{2}}{4 M^{2}}\right) \cos ^{2}(\theta / 2)-\frac{q^{2}}{2 M^{2}}(1+\kappa)^{2} \sin ^{2}(\theta / 2)\right]
\end{aligned}
$$

We obtain the numerical value of this diffusion coefficient by substituting the constants in the transport cross section. Once we have the diffusion coefficients, we numerically solve the diffusion equation in the FLRW metric. In this case, we use the same program but double the stepsize to accommodate the larger radius of the horizon.

As mentioned before we are considering inhomogeneities whose sizes are in the range of $1 \mathrm{~km}$. Since the horizon size is around $10 \mathrm{kms}$ in the hadronic phase, these are large inhomogeneities. We have considered high amplitudes of the order of $10^{14}$ as well as smaller amplitudes, we find that the decay rate does not depend significantly on the amplitudes. However, we find that in an expanding universe the overdensity falls far more rapidly than in an non-expanding universe. We have shown both the cases in Fig. 7. for comparison. We have checked for the decay separately in the range $200-100 \mathrm{MeV}$ as the muon is still present in the plasma at these temperatures. At lower temperatures the muon density in the plasma becomes negligible.

It seems that large inhomogeneities do decay significantly in an expanding universe but as long as they have very large amplitude, they may still survive upto the nucleosynthesis epoch. So an inhomogeneity whose amplitude is of the order of $10^{8}$ will be decreased to an amplitude of the order of $10^{7}$. Hence inhomogeneities with low amplitudes of the order of 10 will be wiped out. Finally, we look at the temperature range from 100 to $1 \mathrm{MeV}$. The muons will be negligible in this epoch but the diffusion coefficients will not change. Figure 8 shows the decay of the inhomogeneities in this epoch.

We find that the amplitude of the inhomogeneity decreases by an order of $10^{4}$ in this period. This means that any inhomogeneity with an amplitude less that $10^{4}$ will be wiped out

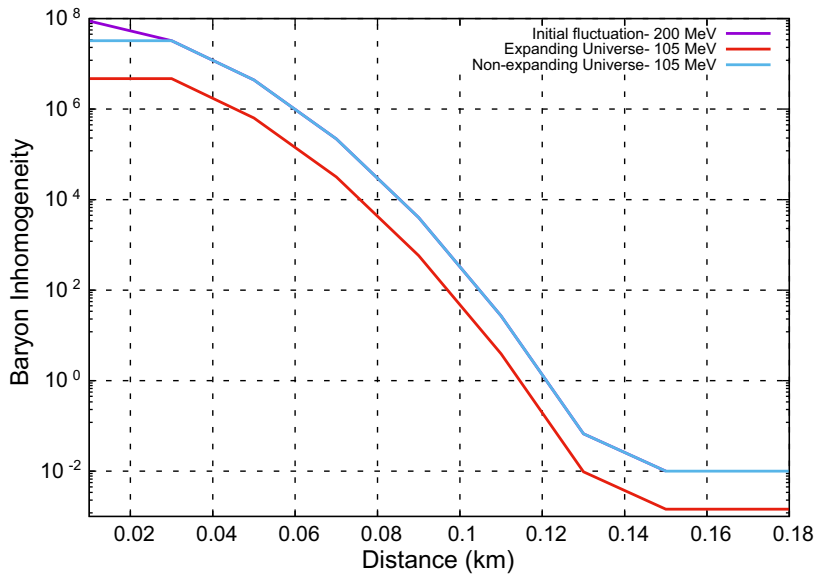

Fig. 7 The decay of the initial fluctuation is shown in logscale between 200 and $100 \mathrm{MeV}$

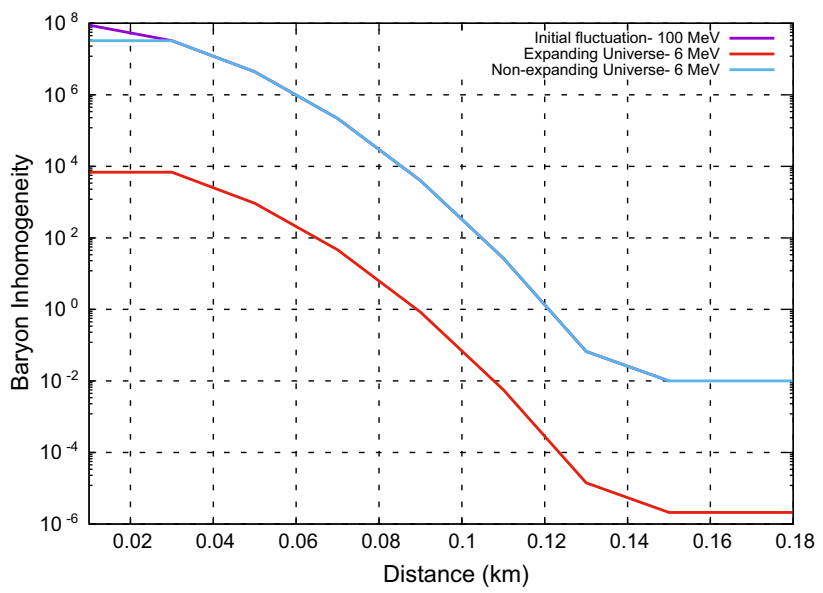

Fig. 8 The decay of the initial fluctuation is shown in logscale between 100 and $1 \mathrm{MeV}$

before the nucleosynthesis epoch. So large baryon inhomogeneities generated during the quark hadron transition must have amplitudes greater than $10^{5}$ times the background density to survive till the nucleosynthesis epoch.

\section{Summary}

In summary, we have done a detailed study of the decay of the baryon inhomogeneities generated at the electroweak scale. Baryon inhomogeneities have important consequences in the early universe. If they survive till the quark hadron phase transition they will affect the phase transition dynamics. The quark hadron phase transition is very important in the thermal history of the universe. Moreover, baryon inhomogeneities can also be generated during the quark hadron phase transition. These will have an effect on the Big Bang Nucleosynthesis calculations. Thus the decay of baryon inhomogeneities are important in the early universe. There has been no pre- 
vious studies of the decay of baryon inhomogeneities in the early universe during the electroweak scale. We have studied the decay of these inhomogeneities in the presence of electrons, muons and neutrinos. The baryon number is carried by the quarks at these high temperatures, so as the inhomogeneity decays, the quarks diffuse through the electrons, muons and neutrinos. The diffusion coefficients for the different particle interactions are calculated. We then use these diffusion coefficients to study the diffusion of the baryon inhomogeneity using the diffusion equation in the FLRW metric.

We have found that baryon inhomogeneities generated in the electroweak epoch should have an amplitude greater than $10^{5}$ for them to survive till the quark hadron phase transition. This makes it difficult for the baryon inhomogeneities generated in a first order electroweak phase transition to have any effect on the quark hadron epoch. In Refs. [5,6], spherical inhomogeneities with a radial profile are formed at the electroweak phase transition. However, the amplitude of these inhomogeneities are bounded by the ratio of the highest and lowest wall velocities reached during the bubble expansion phase. In most cases, this is of the order of $10^{2}$. This is an order of magnitude lower than the amplitude of the baryon inhomogeneities we have considered in our simulations. Thus the baryon inhomogeneities generated due to a strong first order electroweak phase transition would be completely wiped out before the QCD phase transition. To survive upto the QCD phase transition, the baryon inhomogeneities have to have a very high amplitude as they decay substantially during the period between $200 \mathrm{GeV}$ and 200 $\mathrm{MeV}$. This is because the diffusion coefficient is temperature dependent. The quark hadron transition occurs around $200 \mathrm{MeV}$. We have found that the amplitude of the baryon inhomogeneity decreases to about five orders of magnitude during this period. This means any inhomogeneity with an amplitude of $10^{5}$ (or less) will be wiped away before the quark hadron phase transition. We therefore conclude that any model which generates inhomogeneities with less than $10^{5}$ amplitude in the electroweak epoch cannot affect the quark hadron phase transition. They will therefore not contribute to inhomogeneous BBN either.

In this work, due to the vast differences in the values of the diffusion coefficients of the different particles in the electroweak epoch, we have not considered multi particle diffusion. We have studied the diffusion of the quarks through a gas of similar particles only. However, for all the different particles we find that the inhomogeneities will decay. This indicates that even for a multi particle diffusion our results will hold. However, in any region which has a predominance of neutrinos and electrons, the inhomogeneities will probably decay faster, than in regions which have a predominance of muons.

Finally in a previous work, we had looked at the decay of baryon inhomogeneities in the QCD epoch for a station- ary universe. This would work only for small scale inhomogeneities for which the expansion of the horizon does not matter. We have extended that work for an expanding universe where we can work with large baryon inhomogeneities. So we look at the decay of large baryon inhomogeneities in the QCD epoch. We find that the baryon inhomogeneities decrease by $5-6$ orders of magnitude. This means that if large baryon inhomogeneities are generated by collapsing domain walls and other topological defects during the quark hadron transition they will survive till the nucleosynthesis epoch. We conclude that the big bang nucleosynthesis, can thus be used to constrain models which generate large amplitude inhomogeneities in the QCD epoch only.

Acknowledgements The authors acknowledge discussions with Soumen Nayak and Salil Joshi. A.S is supported by the INSPIRE Fellowship of the Department of Science and Technology (DST) Govt. of India, through Grant no: IF170627. The authors would like to thank the anonymous referee for the constructive comments.

Data Availability Statement This manuscript has no associated data or the data will not be deposited. [Authors' comment: The paper is a simulation of the diffusion equation in the expanding universe. The code is available with the authors and can be shared with others on request. All the results of the paper are reproducible using the simulation.]

Open Access This article is licensed under a Creative Commons Attribution 4.0 International License, which permits use, sharing, adaptation, distribution and reproduction in any medium or format, as long as you give appropriate credit to the original author(s) and the source, provide a link to the Creative Commons licence, and indicate if changes were made. The images or other third party material in this article are included in the article's Creative Commons licence, unless indicated otherwise in a credit line to the material. If material is not included in the article's Creative Commons licence and your intended use is not permitted by statutory regulation or exceeds the permitted use, you will need to obtain permission directly from the copyright holder. To view a copy of this licence, visit http://creativecomm ons.org/licenses/by/4.0/.

Funded by SCOAP $^{3}$.

\section{References}

1. E.M. Lifschitz, Physics (Moscow) 10, 116 (1946)

2. S.W. Hawking, Astrophys. J. 145, 544 (1966)

3. J.M. Bardeen, Phys. Rev. D 22, 1882 (1980)

4. A.F. Heckler, Phys. Rev. D 51, 405 (1995)

5. A. Megevand, F. Astorga, Phys. Rev. D 71, 023502 (2005)

6. A. Megevand, A.D. Sanchez, Phys. Rev. D 77, 063519 (2008)

7. G.M. Fuller, G.J. Mathews, C.R. Alcock, Phys. Rev. D 37, 1380 (1988)

8. H. Kurki-Suonio, Nucl. Phys. B Proc. Suppl. 24(2), 67-73 (1991)

9. B. Layek, S. Sanyal, A.M. Srivastava, Phys. Rev. D 67, 083508 (2003)

10. A. Cohen, D. Kaplan, A. Nelson, Phys. Lett. B 245, 561 (1990)

11. A. Cohen, D. Kaplan, A. Nels, Nucl. Phys. B 349, 727 (1991)

12. A. Cohen, D. Kaplan, A. Nelson, Phys. Lett. B 263, 86 (1991)

13. L.D. McLerran, M.E. Shaposhnikov, N. Turok, M.B. Voloshin, Phys. Lett. B 256, 451 (1991)

14. L.D. McLerran, Phys. Rev. Lett. 62, 1075 (1989) 
15. M.E. Shaposhnikov, JETP Lett. 44, 465 (1986)

16. M.E. Sha-poshnikov, Nucl. Phys. B 287, 757 (1987)

17. M.E. Shaposhnikov, Nucl. Phys. B 299, 797 (1988)

18. N. Turok, P. Zadrozny, Phys. Rev. Lett. 65, 2331 (1990)

19. N. Turok, P. Zadrozny, Nucl. Phys. B 358, 471 (1991)

20. S. Sanyal, Phys. Rev. D 67, 074009 (2003)

21. K. Jedamzik, G.M. Fuller, Astrophys. J. 423, 33-49 (1994)

22. N. Sasaki, O. Miyamura, S. Muroya, C. Nonaka, Phys. Rev. C 62(1), 011901 (2000)

23. R.J. Scherrer, M.S. Turner, Phys. Rev. D 100(4), 043545 (2019)

24. S. Sau, S. Bhattacharya, S. Sanyal, Eur. Phys. J. C 79(5), 439 (2019)

25. B. Layek, S. Sanyal, A.M. Srivastava, Phys. Rev. D 63, 083512 (2001)

26. S. Cherubini, P. Figuera, A. Musumarra et al., Eur. Phys. J. A 20, 355-358 (2004)

27. A. Arbey, J. Auffinger, J. Silk, Phys. Rev. D 102, 023503 (2020)

28. J.H. Applegate, C.J. Hogan, R.J. Scherrer, Phys. Rev. D 35, 11511160 (1987)

29. I.-S. Suh, G.J. Mathews, Phys. Rev. D 58, 123002 (1998)

30. R. Nakamura, M. Hashimoto, R. Ichimasa, K. Arai, Int. J. Mod. Phys. E 26(08), 1741003 (2017)

31. K. Inomata, M. Kawasaki, A. Kusenko, L. Yang, J. Cosmol. Astrophys. 12, 003 (2018)

32. J.D. Barrow, R.J. Scherrer, Phys. Rev. D 98(4), 043534 (2018)

33. J. Dziarmaga, Phys. Rev. D 52, R569 (1995)
34. M. Nagasawa, Astropart. Phys. 5, 231 (1996)

35. M. Sato, Phys. Lett. B 376, 41 (1996)

36. M. Nagasawa, J. Yokoyama, Phys. Rev. Lett. 77, 2166 (1996)

37. H.K. Lo, Phys. Rev. D 51, 7152 (1995)

38. M. Barriola, Phys. Rev. D 51, R300 (1995)

39. R.H. Brandenberger, A. Riotto, Phys. Lett. B 445, 323 (1999)

40. T. Matsuda, Phys. Rev. D 64, 083512 (2001)

41. A. Ray, S. Sanyal, Phys. Lett. B 726(1-3), 83-87 (2013)

42. A. Atreya, A. Sarkar, A.M. Srivastava, J. Phys. Conf. Ser. 484, 012053 (2012)

43. A. Atreya, A. Sarkar, A.M. Srivastava, Phys. Rev. D 90(4), 045010 (2014)

44. Michael B. Christiansen, Jes Madsen, Phys. Rev. D 53, 5446-5454 (1996)

45. L.D. Landau, E.M. Lifshitz, Physical Kinetics (Pergamon Press Ltd., Oxford, 1981)

46. K.S. McFarland, Nucl. Phys. B Proc. Suppl. 235, 143-148 (2013)

47. D.J. Schwarz, Ann. Phys. 12, 220-270 (2003) 